\title{
Isodicentric Ychromosome: Case Study
}

\author{
Amal Al Wathnani* ${ }^{\circledR}$, Suha Tashkandi, Khelad Al Saidi, Kholoud Al Muteiri, Wasmaya Al Enezi \\ Pathology and Clinical Laboratory Medicine Administration, Cytogenetic Department, King Fahad Medical City, Riyadh, \\ Saudi Arabia \\ Email: ^aalwathnani@kfmc.med.sa, stashkandi@kfmc.med.sa,kalsaidi@kfmc.med.sa, \\ kalmuteiri@kfmc.med.sa.walanazi@kfmc.med.sa
}

How to cite this paper: Al Wathnani, A., Tashkandi, S., Al Saidi, K., Al Muteiri, K. and Al Enezi, W. (2021) Isodicentric Ychromosome: Case Study. Open Journal of Genetics, 11, 56-62.

https://doi.org/10.4236/ojgen.2021.113006

Received: March 14, 2020

Accepted: September 27, 2021

Published: September 30, 2021

Copyright $\odot 2021$ by author(s) and Scientific Research Publishing Inc. This work is licensed under the Creative Commons Attribution International License (CC BY 4.0).

http://creativecommons.org/licenses/by/4.0/

\begin{abstract}
Isodicentric chromosomes are a frequently appearing abnormality in the human Y chromosome. Making predictions regarding the phenotypic outcomes of a variety of duplications/deletions in the dicentric $\mathrm{Y}$ chromosome generally depends on the breakpoint location and also the level of mosaicism (45, X cell lines); in certain instances, these may not be detected and result in variations running between male, abnormal female, or ambiguity in individual genitalia. A referral was received from a urology clinic concerning two patients, one aged 34 and one aged 35, with a request to investigate reasons for infertility: G-banded karyotyping and fluorescence in situ hybridization (FISH) revealed the presence of an isodicentric $\mathrm{Y}$ chromosome.
\end{abstract}

\section{Keywords}

Isodicentric Y, Mosaicism, Fluorescence in Situ Hybridization, SRY, G-Banded Karyotyping, Infertility

\section{Introduction}

Chromosome Y plays an important part in fertilization and development for males because it holds the SRY gene that determines sex and numerous spermatogenesis genes, e.g. DAZ, AZF1/2 [1]. Various forms of abnormality can be found in the $\mathrm{Y}$ chromosome; these are divided into numerical or structural abnormalities. One of the most frequently found structural abnormalities with $\mathrm{Y}$ chromosomes is the isodicentric (idic) Y chromosomes [2]. It is characteristic of this abnormality to present with a pair of identical arms, mirroring each other through positioning by the two centromeres [3]. It has been shown [1] [4] that the idic Y chromosome is created as part of cell division when opposite arms of sister chromatids undergo homologous crossing. The process commences with a double-sided breakpoint in one of the Y chromosome's arms and a subsequent 
homologous repair which employs the opposite arm for a template [4] [5]. From this we can see that idic $(\mathrm{Y})$ chromosome abnormalities arise from defects as a part of gametogenesis before the spermatid stage because two chromatids are required to create such structural abnormalities, occurring from errors at the initial zygotic division. If an error were to occur following the first zygotic division, mosaicism would be the outcome [4] (Figure 1).

Because of this, idic Y chromosomes depend on where breakpoints opposition. If the breakpoint in an idic Y chromosome occurs in the short arm (p), it will be a duplicate of the whole long arm (q) and the proximal short arm and so will be classified as idic Yq. However, if the breakpoint occurs in the long arm, it will duplicate the whole short arm and the proximal long arm, and will be identified as idic Yp [3]. Because two centromeres are present, there is frequently instability in the abnormal chromosome in the cell division process [1].

Resulting from this, two cell lines (i.e., mosaicism) are frequently found, with the majority of patients having a $45, \mathrm{X}$ cell line [5]. Degrees of mosaicism vary considerably and variations may even be found within a patient's cell lines [6] [6]. Furthermore, such mosaic karyotypes have associations with a variety of clinical features, e.g., gonadal dysgenesis and Turner's syndrome [7]. Clinical phenotype severity is dependent on where the breakpoints occur and also particular cell lines' degree and tissue distribution, particularly 45, X [8]. Breakpoints in the chromosome Y's long arm may cause deletions or rearrangements of critical azoospermia factor (AZF) regions; these are regarded as the most significant breakpoints [6]. Any deletion within AZF regions may cause differing levels of spermatogenic failure, and this has been suggested as the reason for fertility in patients with idicenteric chromosome $\mathrm{Y}$.

Healthy male patients who have chromosomal abnormalities are candidates for infertility, especially if they have abnormalities in the $\mathrm{Y}$ chromosome demonstrating azoospermia (no sperm) severe oligozoospermia $\left(<1 \times 10^{6} \mathrm{sperm} / \mathrm{ml}\right.$ semen), moderate oligozoospermia $\left((1-5) \times 10^{5} \mathrm{sperm} / \mathrm{ml} / \mathrm{semen}\right)$ or mild oligozoospermia $\left((5-20) \times 10^{5} \mathrm{sperm} / \mathrm{ml}\right.$ semen $)$ [2]. There are associations between $\mathrm{Y}$

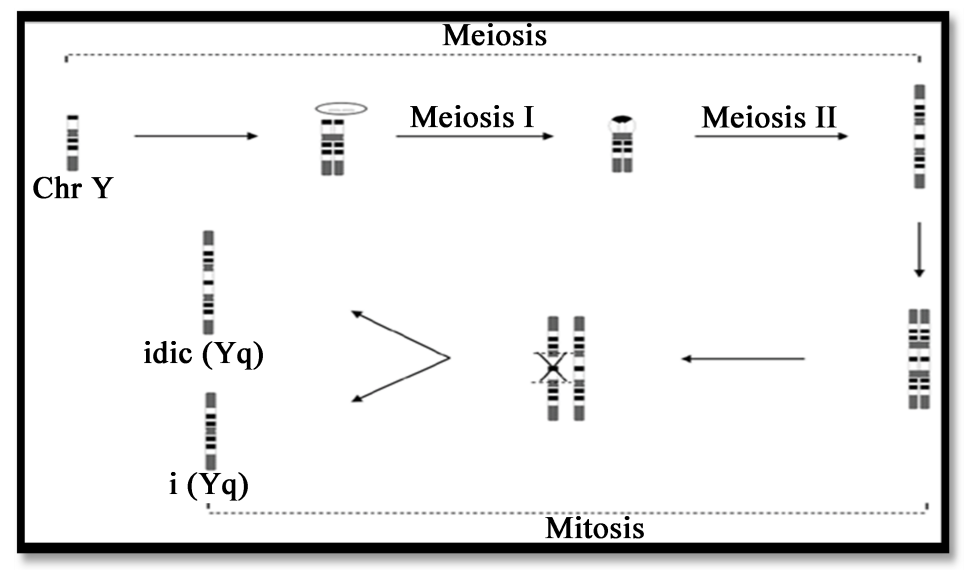

Figure 1. Schematic diagram of origin mechanism of idic (Yq) and i (Yq). (DOI: https://doi.org/10.3892/mmr.2017.6981 ) 
chromosome deletions and oligozoospermia. Chromosome abnormalities can be found using routine karyotyping and FISH or microarray techniques, with chromosome abnormalities being found in between $5 \%$ and $10 \%$ of such subjects. Molecular genetic tests show that between 5\% and 13\% of such subjects have microdeletions within the Y chromosome's long arm [9]. Although we have detailed molecular information regarding both the controlling regions for spermatogenesis and identifying the genes that are part of the $\mathrm{Y}$ chromosome [8], it has yet to be conclusively shown that sterility found in subjects with isodicentric $\mathrm{Y}$ chromosomes is attributable to abnormalities in the chromosome that could disrupt accurate heterochromosome pairing or if it is attributable to contemporary deletion in the AZF regions that are crucial for spermatogenesis [2] [7].

\section{Case Presentations}

A urology clinic referred two patients, one aged 34 and one aged 35, for infertility investigations. Azoospermia with normal hormone markers was detected by recent semen analysis.

Cytogenetic investigations were undertaken with informed agreement on the peripheral blood lymphocytes employing Fluorescence In Situ Hybridisation (FISH) and G-banding techniques. FISH investigations were undertaken with the same samples for confirmation of the abnormality, employing these FISH probes:

- Vysis CEP X SpectrumGreen (Xp11.1-q11.1 alpha satellite DNA)/CEPY (DYZ3) SpectrumOrange (Yp11.1-q11.1 alpha satellite DNA) Vysis Probe Kit.

- LSI SRY SpectrumOrange (Yp11.3)/CEP X (DXZ1) SpectrumGreen (Xp11.1q11.1) Probe Kit (Abbott Molecular Inc., Des Plaines, IL, USA) as per manufacturer instructions.

- Multi-color DNA probe mixtures were employed for identification of the subtelomeric regions from the short (Yp) and the long arm (Yq) of the $\mathrm{Y}$ chromosome (ToTelVysion, USA).

A 20 metaphases sample was analyzed using GTG banding (550 bands resolution) which revealed a mosaicism 46, XY, idic (Y) (q11.21) [10]/45, X [10], and 46, XY, idic (Y)(q11.21) [12]/45, X [8] respectively. So the Y chromosome, if present, was small in size in comparison to normal samples (Figure 2).

Additionally, the analysis of 500 interphases employing FISH techniques using fluorescent microscopy indicated that two centromere regions were present on the Y chromosome; two SRY genes (Yp11.3) were also found that indicated that the rearranged $Y$ chromosome had two short arms (Figure 3).

Other FISH probes, employing particular probes to investigate the telomeric regions in the $\mathrm{Y}$ chromosome, supported these observations. With telomere Yp-specific probes, we found a pair of fusion signals that corresponded with the telomeric regions of the $\mathrm{Y}$ chromosome's short arms (Figure 4). Chromosome 1 was employed as an internal control in this field. Telomere probing of the $\mathrm{Y}$ 


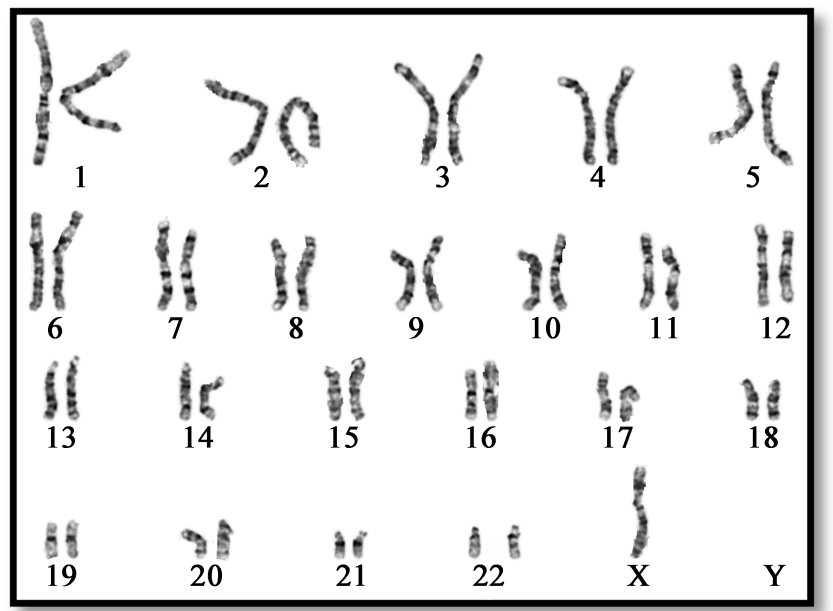

(a)

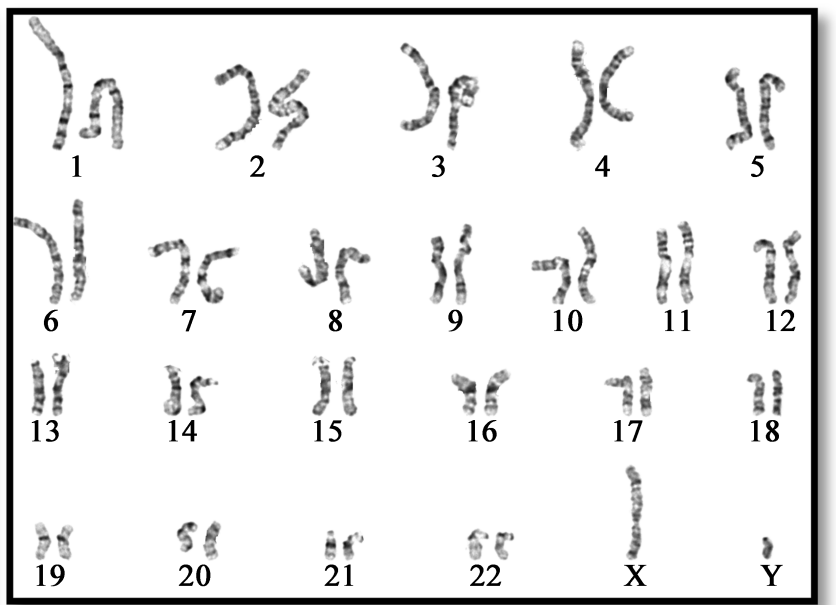

(b)

Figure 2. Karyotype of the patient found with GTG-banding, showing (a) a 45, X cell line and (b) a 46, XY, idic (Y) (q11.21) if present, was small in size in comparison to normal samples.

(a)

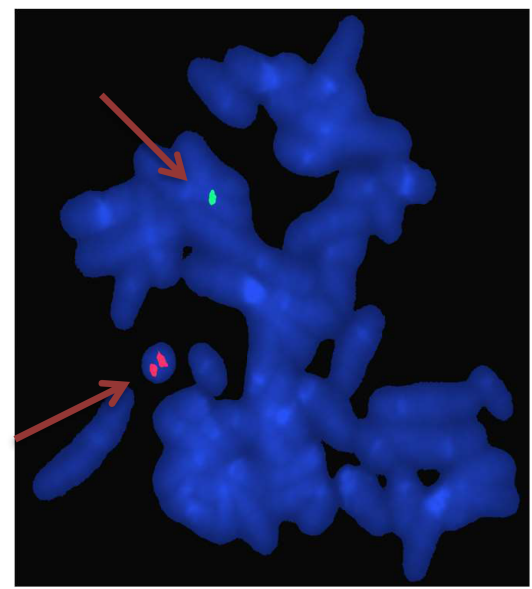

(c)

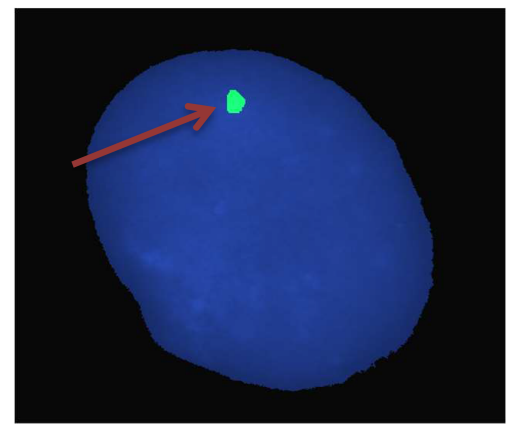

(b)

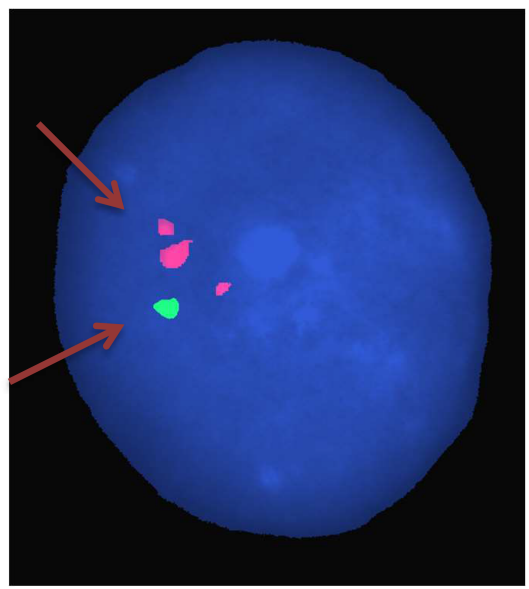

(d)

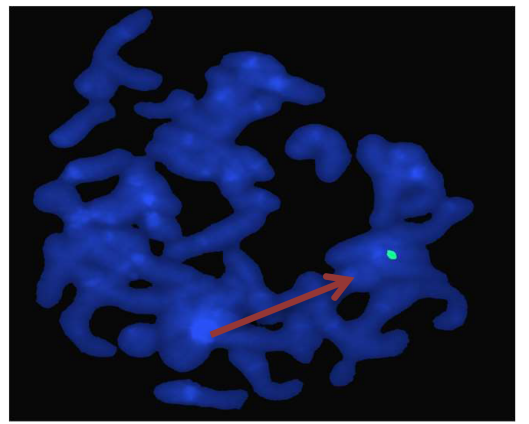

Figure 3. Fluorescent in situ hybridization employing centromeric X probe (CEP X SpectrumGreen hybridizes to the centromere of human X chromosome), LSI SRY probe, specific to the SRY gene (Yp 11.3) and for centromeric Y sequence DYZ3 (CEP Y spectrum orange hybridizes to the centromere of human chromosome $\mathrm{Y}$ ): (a) Metaphase displaying two orange signals were found, indicating that the re-arranged Y chromosome held 2 SRY genes and 2 short arms; the green signal shows CEP X (Xp11.1-q11.1). (b) FISH undertaken with the mix probe employing three probes on the interphase. 1) Alpha satellite probe, region-specific (including the centromere) with a span from Yp11.1 to Y q11, showed the existence of 2 centromeric structures in the Y chromosome. 2) LSI SRY probe, SRY gene-specific (Yp 11.3) is representative of 2 short arms of chromosome Y. 3) CEP X (Xp11.1-q11.1), one green signal shows chromosome X centromere. 4) Both a and b represent 46, XY, idic (Y) (q11.21) cell line. (c) Interphase displaying one green signal for the $\mathrm{X}$ chromosome and no red signals for the $\mathrm{Y}$ chromosome. (d) Metaphase displaying one green signal for the $\mathrm{X}$ chromosome and no red signals for the $\mathrm{Y}$ chromosome. Both $\mathrm{C}$ and $\mathrm{d}$ represent 45 , X cell line. 
chromosome's Yq found nothing. In this field chromosome 2 was employed as an internal control.

Because of this, it was demonstrated that the idic Yq has breakpoints distal to the AZFc region, probably within the distal Yq pseudoautosomal region 2 (PSR2) (Figure 5). Y-chromosome abnormalities were caused by duplication of the whole short arm ( $\mathrm{p}$ arm) and also virtually the whole of the long arm ( $\mathrm{q}$ arm) for the $\mathrm{Y}$ chromosome with two matching facing centromeres and a deletion in the PSR2 region.

\section{Discussion/Conclusions}

The $\mathrm{Y}$ chromosome is a crucial part of fertilization, and around $6 \%$ of infertile males have structural chromosome abnormalities, e.g., duplications, deletions, inversions, dicentrics, and rings; in subjects with azoospermia, this number rises

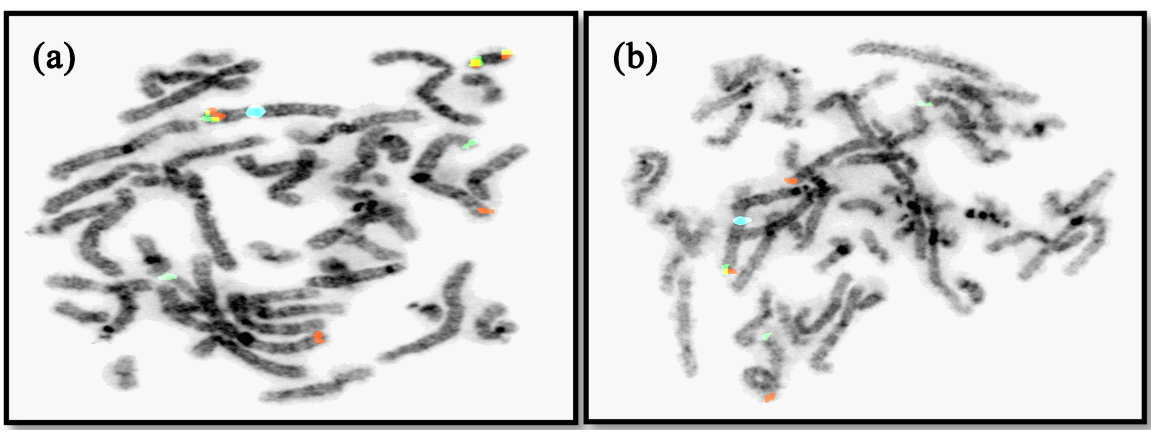

Figure 4. (a) With Yp specific FISH probes, three fusion signals were found, with two fusions that represent the telomeric regions of the Y chromosome's short arms; in the same field $1 \mathrm{p}$ (green) and $1 \mathrm{q}$ (red) as well as $\mathrm{Xp}$ (fusion) specific probes were employed for internal controls. (b) The telomeric region of the long arm of the $\mathrm{Y}$ chromosome could not be found with Yq specific FISH probes. In this field 2p (green) and $2 \mathrm{q}$ (red) and also Xq (fusion) specific probes were employed for detection of the corresponding telomeric regions of chromosome 2 and $\mathrm{X}$, as internal control.

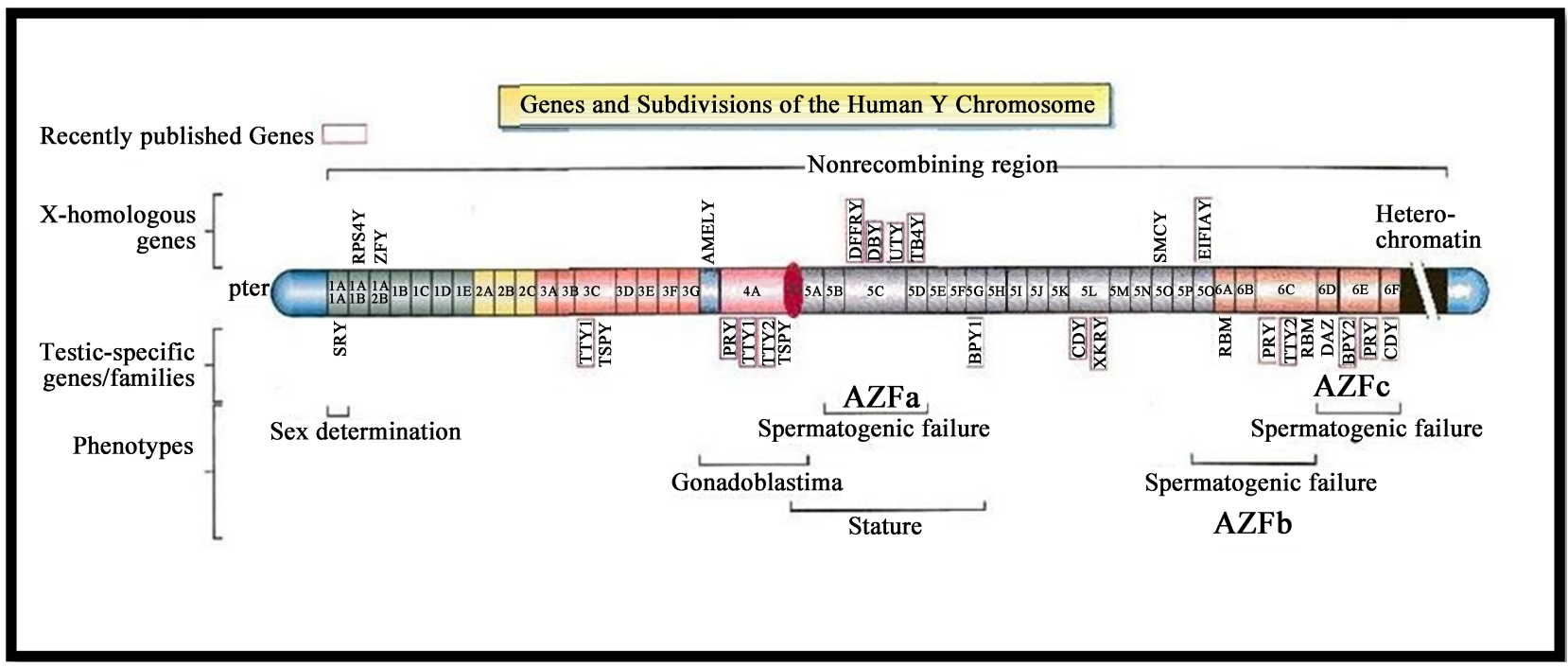

Figure 5. A functional map of Y chromosome (MMG 2332014 Genetics \& Genomics Wiki, 2019). 
to $15 \%$ [6]. The instabilities within mitotically isodicentric Y have associations with Turner syndrome and sex reversal cell lines [7]. Five instances were found where such karyotypes had associations with standard male phenotypes [2]. As already shown, there was a great variety of phenotypes in subjects with idic $\mathrm{Y}$ chromosome, in a range from males with infertility to Turner-light females, depending on dicentric Y chromosome structure, types of mosaicism, and Yp and Yq breakpoints. $40.9 \%$ of subjects were phenotypical females, 31.8\% phenotypical males, and $27.3 \%$ had varying levels of intersexuality [6].

This research details two instances in 2018 and 2019 shown to be a mosaic with the characterizing karyotype: mos 46, XY, idic (Y) (q11.21) [10]/45, X [10], and 46, XY, idic (Y) (q11.21) [12]/45, X [8]. With these instances, the Y chromosome is isodicentric which translates as a deletion inside the $\mathrm{q}$ arm beginning at the q11.21 region. Additional characterization is that the two regions of $\mathrm{AZF} 2$, containing the $\mathrm{AZFb}$ at the 11.22 band and the AZFc at 12 band, are absent. There are more associations with serious infertility when the AFZb region is completely deleted than with the deletion of the AZFc region, so AZFb deletion is associated with a negative prognosis. When the AZFc region is deleted there is still a $50 \%$ chance of spermatozoa being detected within the testicular tissue [11]. Azoospermia in the subject may be attributable to the existence of micro-deletions, particularly concerning the AZF2 and DAZ (Yq11.21) genes. Several researchers into infertility have recently emphasized the key role in spermatogenesis played by AZF regions [8]. This is due to the fact that they contain a minimum of 16 genes that play important roles in spermatogenesis, and various levels of male factor infertility can be caused by deletions in these areas [1].

It is hoped that in our future research we can undertake deeper analysis, looking at micro-deletions within the $\mathrm{Y}$ chromosome through PCR for detection of those regions in the AZF area that have been deleted.

\section{Availability of Data}

The data analysed during this study are available with the authors.

\section{Acknowledgements}

I would like to thank the research center, King Fahad Medical City Riyadh, Saudi Arabia, for their help in publishing the manuscript.

\section{Authors' Contributions}

AS drafted the article and revised it critically for important intellectual content. $\mathrm{KS}, \mathrm{KM}$ and WE substantial contributed to analysis and acquisition of results of cases. ST analyzed and interpreted the data.

\section{Conflicts of Interest}

The authors declare no conflicts of interest regarding the publication of this paper. 


\section{References}

[1] Lehmann, K., Kovac, J., Xu, J. and Fischer, M. (2012) Isodicentric Yq Mosaicism Presenting as Infertility and Maturation Arrest without Altered SRY and AZF Regions. Journal of Assisted Reproduction and Genetics, 29, 939-942. https://doi.org/10.1007/s10815-012-9822-y

[2] Colao, E., Vismara, M.F.M., Bombardiere, F., Fabiani, F. and Grillone, T. (2015) Clinical and Molecular Evaluation of a Case of Male Infertility and Azoospermia. Journal of Case Reports and Studies, 3, 102.

[3] Bergeron, M., Brochu, P., Lemyre, E. and Lemieux, N. (2011) Correlation of Intercentromeric Distance, Mosaicism, and Sexual Phenotype: Molecular Localization of Breakpoints in Isodicentric Y Chromosomes. American Journal of Medical Genetics Part A, 155, 2705-2712. https://doi.org/10.1002/ajmg.a.34260

[4] Lange, J., Skaletsky, H., van Daalen, S., Embry, S., Korver, C., Brown, L., Oates, R., Silber, S., Repping, S. and Page, D. (2009) Isodicentric Y Chromosomes and Sex Disorders as Byproducts of Homologous Recombination That Maintains Palindromes. Cell, 138, 855-869. https://doi.org/10.1016/j.cell.2009.07.042

[5] Becker, R. and Akhavan, A. (2016) Prophylactic Bilateral Gonadectomy for Ovotesticular Disorder of Sex Development in a Patient with Mosaic 45,X/46, X,idic(Y)q11.222 Karyotype. Urology Case Reports, 5, 13-16. https://doi.org/10.1016/j.eucr.2015.12.003

[6] Jiang, Y., Wang, R., Li, L., Xue, L., Deng, S. and Liu, R. (2017) Molecular-Cytogenetic Study of de Novo Mosaic Karyotype 45,X/46,X,i(Yq)/46,X,idic(Yq) in an Azoospermic Male: Case Report and Literature Review. Molecular Medicine Reports, 16, 3433-3438. https://doi.org/10.3892/mmr.2017.6981

[7] Trombetta, B. and Cruciani, F. (2017) Y Chromosome Palindromes and Gene Conversion. Human Genetics, 136, 605-619. https://doi.org/10.1007/s00439-017-1777-8

[8] Lee, J., Park, J., Kim, D., Lee, H., Choi, S. and Cho, Y. (2019) Detailed Analysis of Isodicentric $\mathrm{Y}$ in a Case with Azoospermia and 45,X/46,X,Idic(Y) Mosaicism. http://www.annclinlabsci.org/content/45/2/206.long

[9] Silber, S. and Disteche, C. (2019) Y Chromosome Infertility. https://www.ncbi.nlm.nih.gov/books/NBK1339/

[10] MMG 2332014 Genetics \& Genomics Wiki. (2019) The Loss of the Y Chromosome. https://mmg-233-2014-genetics-genomics.fandom.com/wiki/The_Loss_of_the_Y_C hromosome

[11] Bansal, S., Jaiswal, D., Gupta, N., Singh, K., Dada, R., Sankhwar, S., Gupta, G. and Rajender, S. (2016) Gr/gr Deletions on Y-Chromosome Correlate with Male Infertility: An Original Study, Meta-Analyses and Trial Sequential Analyses. Scientific Reports, 6, Article No. 19798. https://doi.org/10.1038/srep19798 